\title{
Foot drop after a suicide attempt
}

\author{
Atul K. Taneja • Laercio A. Rosemberg • \\ Alexandre O. Kaup
}

Published online: 30 May 2014

(C) ISS 2014

Keywords Sciatic $\cdot$ Neuropathy $\cdot$ Denervation Myonecrosis · MRI · Drop foot

\section{Sciatic neuropathy with denervation and myonecrosis}

A 33-year-old male presented with a 2-month history of left foot drop, starting upon waking up 3 days after a suicide attempt with ingestion of high doses of phenobarbital, clonazepam, and alcohol. Painful left drop foot, trophic changes in the lower extremities, and bilateral Achilles areflexia were noted. Magnetic resonance neurography (MRN) of the lumbosacral plexus and left thigh showed thickening and signal intensity (SI) abnormality in the left sciatic nerve along the pelvic outlet and proximal thigh (Fig. 1). Denervation of the lumbosacral plexus affecting the quadratus femoris, piriformis, obturator, and adductor muscles with areas without contrast enhancement consistent with myonecrosis was seen on

No related papers from the same study have been published or submitted.

The case presentation can be found at doi:10.1007/s00256-014-1904-8

A. K. Taneja $\cdot$ L. A. Rosemberg

Musculoskeletal Radiology Division, Imaging Department, Hospital

Israelita Albert Einstein, São Paulo, Brazil

A. O. Kaup

Neurology Department, Hospital Israelita Albert Einstein, São Paulo, Brazil

\author{
A. K. Taneja $(\square)$ \\ Departamento de Imagem-Hospital Israelita Albert Einstein, Av. \\ Albert Einstein, 627, Morumbi, São Paulo, SP, Brazil CEP \\ 05652-900 \\ e-mail: taneja@einstein.br
}

the pelvis, while denervation of the hamstring muscles related to sciatic neuropathy was seen on the thigh (Fig. 2). Electroneuromyography showed complete motor and sensitive axonal impairment of the left peroneal nerve, partial impairment of the left tibial nerve (with reinnervation), and partial impairment of the left femoral nerve.

A compressive-ischemic mechanism after a long-term sideways position during unconsciousness was indicated as the cause of the clinical and imaging findings in this case. Sciatic neuropathy is the second most common cause in the legs, after peroneal neuropathy, and may be caused by acute or chronic compression, stretching, ischemia, laceration, or toxic injury (injections) as well as iatrogenic lesions (hip arthroplasty). Increased pressure on the nerve can cause injury either directly or indirectly as a result of compression of the vasa nervorum if the ischemia is prolonged, which was the most likely mechanism in our case [1,2]. The sciatic nerve derives from the fourth and fifth lumbar and first and second sacral roots, coursing down deeper to the piriformis muscle with anatomical variations. It continues posteromedially to the hip and distally deep in the thigh before dividing into the tibial and common peroneal nerves above the popliteal fossa $[2,3]$.

MR imaging patterns vary depending on the muscle denervation stage: acute and subacutely denervated muscles show high SI on fluid-sensitive images and normal SI on T1-weighted images; in chronic denervation, muscle atrophy and fatty infiltration demonstrate high SI on T1-weighted images in association with volume loss [4]. On the other hand, myonecrosis is revealed by high SI on T1-weighted images, heterogeneously high SI on T2weighted images, and rim enhancement on contrastenhanced images [5]. A possible overlap of denervation 

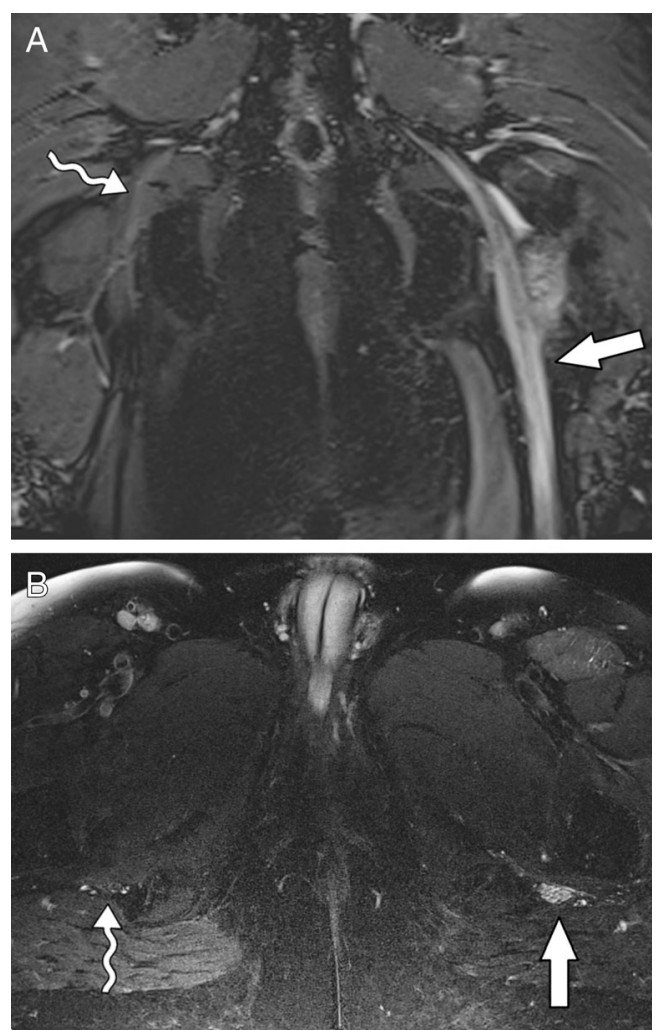

Fig. 1 Coronal (a) and axial (b) SPAIR MRNs of the pelvis show diffuse thickening and abnormal SI of the left sciatic nerve at the pelvis (straight arrows). Normal sciatic nerve on the right side is demonstrated (wavy arrow) changes with ischemic myonecrosis due to prolonged extrinsic pressure should be considered for the muscle abnormalities found on MRN as well as sequela of rhabdomyolysis from electrolyte imbalance or induced by drugs, even though the time elapsed since the suicide attempt makes this explanation less plausible.

Time until recovery, if recovery occurs, depends on the degree of nerve lesioning, e.g., neurotmesis or neuroapraxia, and may take months to years [6]. MRN has significant potential to identify plexus injuries and nerve compression [7] and plays a fundamental role in clinically and electrodiagnostically inconclusive cases $[8$, 9]. Electrodiagnostic study is time consuming and may provide indeterminate results, especially in postoperative cases; also, it may be uncomfortable or unfeasible in subjects with dermatologic diseases or deeply situated nerves. Moreover, it may not reveal the exact site of nerve injury and is unable to distinguish among adhesive (perineural) fibrosis, neuromas, and compressive mass lesions. In this setting, MRN confirms clinical suspicion by showing nerve abnormality or muscle denervation, assessing the extent of nerve injuries or disease load in diffuse peripheral neuropathies and depicting lesions causing nerve entrapment or impingement. In addition, MRN detects lesions that mimic neuropathy symptoms and provides imaging guidance for perineural injections [9].
Fig. 2 Axial SPAIR (a) and T1weighted fat-suppressed postcontrast (b) MRNs of the left hip show denervation involving the gluteus medius (curved arrow) and myonecrosis affecting the quadratus femoris (arrowhead). Axial T2-weighted fat-suppressed (c) and axial T1-weighted (d) MRNs show denervation and fatty infiltration of the hamstring muscles (curved arrows)
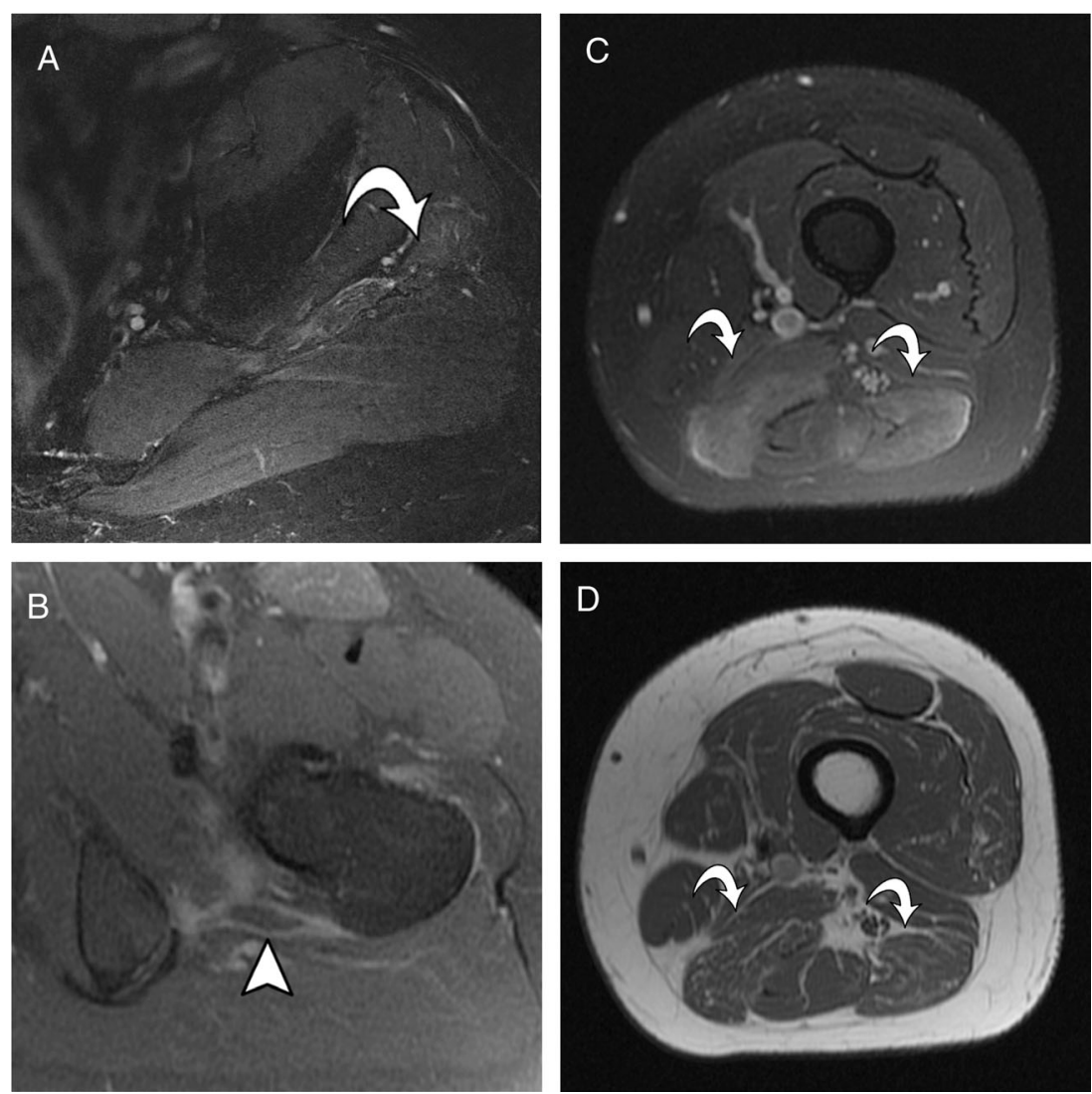
Disclosure No disclosures.

\section{References}

1. Plewnia C, Wallace C, Zochodne D. Traumatic sciatic neuropathy: a novel cause, local experience, and a review of the literature. J Trauma Acute Care Surg. 1999;47(5):986.

2. Yuen EC, So YT. Sciatic neuropathy. Neurol Clin. 1999;17(3): 617-31.

3. Pham M, Wessig C, Brinkhoff J, Reiners K, Stoll G, Bendszus M. MR neurography of sciatic nerve injection injury. J Neurol. 2011;258(6): $1120-5$.

4. Kamath S, Venkatanarasimha N, Walsh MA, Hughes PM. MRI appearance of muscle denervation. Skeletal Radiol. 2007;37(5):397-404.
5. Lu CH, Tsang YM, Yu CW, Wu MZ, Hsu CY, Shih TTF. Rhabdomyolysis: magnetic resonance imaging and computed tomography findings. J Comput Assist Tomogr. 2007;31(3):368.

6. Mumby DM, Hartsilver EL. Magnetic resonance imaging of sciatic nerve compression injury after epidural blockade. Int J Obstet Anesth. 2012;21(2):199-200.

7. Gupta R, Villablanca PJ, Jones NF. Evaluation of an acute nerve compression injury with magnetic resonance neurography. J Hand Surg. 2001;26(6):1093-9.

8. Kim S, Choi J-Y, Huh Y-M, Song H-T, Lee S-A, Kim SM, et al. Role of magnetic resonance imaging in entrapment and compressive neuropathy - what, where, and how to see the peripheral nerves on the musculoskeletal magnetic resonance image: part 1. Overview and lower extremity. Eur Radiol. 2007;17(1):139-49.

9. Chhabra A, Andreisek G, Soldatos T, Wang KC, Flammang AJ, Belzberg AJ, et al. MR neurography: past, present, and future. Am J Roentgenol. 2011;197(3):583-91. 